\title{
Prevalência de polifarmácia, benzodiazepínicos e fatores associados em idosos institucionalizados
}

\author{
Cristine Melania Gatto; Matheus Santos Gomes Jorge"*; Lia Mara Wibelinger"*; Telma Elita Bertolin ${ }^{* * * * *}$ \\ Marilene Rodrigues Portella"*;"; Marlene Doring
}

\section{Resumo}

Com o envelhecimento humano há aumento dos problemas de saúde apresentados pelos idosos e, muitas vezes, os familiares, ou os próprios indivíduos, optam por atendimentos especializados em geriatria, como é o caso das instituições de longa permanência para os idosos. Concomitante a isso, ocorre aumento do consumo de medicamentos por parte desta população, especialmente dos benzodiazepínicos, caracterizando, assim, a polifarmácia. Com base nisto, o objetivo deste estudo foi verificar a prevalência de polifarmácia, benzodiazepínicos e fatores associados em idosos institucionalizados. Estudo transversal, de base populacional, com 219 idosos residentes em dez instituições de longa permanência para idosos distribuídas em Passo Fundo/RS e Bento Gonçalves/RS. Os dados foram coletados por meio de um questionário estruturado, onde registrou-se os dados de identificação, variáveis sociodemográficas, estado cognitivo e condi- ções de saúde. Para análise empregou-se estatística descritiva e inferencial. Observou-se que a maioria da amostra era de mulheres, brancas, com 80 anos ou mais, baixa escolaridade, viúvas, com declínio cognitivo e com doenças crônicas. A prevalência de polifarmácia chegou a 74,5\% e de benzodiazepínicos a 21,1\%. A polifarmácia associou-se à hipertensão arterial sistêmica, demência, depressão e as doenças cardiovasculares $(p<0,05)$, enquanto os benzodiazepínicos associaram-se inversamente ao acidente vascular encefálico e demência $(p<0,05)$. Em suma, as evidências apresentadas podem instigar à construção de políticas públicas, visionadas em melhores condições de saúde e atenção à polifarmácia nesta população.

Palavras-chave: Envelhecimento. Polimedicação. Nível de saúde. Instituição de longa permanência para idosos.

* Farmacêutica. Pós-Graduação em Farmacologia Clínica pela Universidade de Passo Fundo. Mestre em Envelhecimento Humano pela Universidade de Passo Fundo. E-mail: cristinegatto@hotmail.com.

** Fisioterapeuta. Especialização em Fisioterapia Traumato-Ortopédica pela Universidade de Passo Fundo. Mestre em Envelhecimento Humano pela Universidade de Passo Fundo. E-mail: matheussgjorge@gmail.com.

*** Fisioterapeuta. Doutora em Gerontologia Biomédica pela Pontifícia Universidade Católica do Rio Grande do Sul. Pós-doutorado em Gerontologia pela Universidade Estadual de Campinas. Docente do Programa de Pós-Graduação em Envelhecimento Humano da Universidade de Passo Fundo. E-mail: liafisio@upf.br.

***** Bióloga. Doutora em Tecnologia Bioquímico-Farmacêutica Pela Universidade de São Paulo. Pós-doutorado em Neurociências Celular e Molecular pela Universidade de Lisboa, Portugal. Docente do Programa de Pós-Graduação em Ciência e Tecnologia de Alimentos da Universidade de Passo Fundo. E-mail: telma@upf.br.

******* Enfermeira. Doutorado em Enfermagem pela Universidade Federal de Santa Catarina. Docente do Programa de Pós-Graduação em Envelhecimento Humano da Universidade de Passo Fundo. E-mail: portella@upf.br.

${ }^{* * * * * * * *}$ Enfermeira. Doutorado em Enfermagem pela Universidade Federal de Santa Catarina. E-mail: doring@ upf.br.

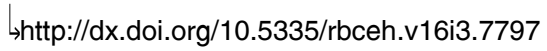




\section{Introdução}

O envelhecimento populacional é um fenômeno global progressivo. Cerca de 15 milhões de brasileiros são idosos, ou seja, possuem 60 anos ou mais (CRUZ et al., 2015; GLENZ et al., 2015), tornando o país o quinto maior do mundo neste quesito. Estimativas indicam que haverá um aumento neste grupo de indivíduos nos próximos 30 anos, atingindo 63 milhões em 2050 (CAMARANO; KANSO, 2010).

Com o aumento da expectativa de vida dos brasileiros, aumenta, também, a ocorrência de doenças crônicas não transmissíveis (PRESTA et al., 2011), dentre as quais pode-se citar a hipertensão arterial sistêmica, o diabetes mellitus, a demência, a insônia, as doenças cardiovasculares, a depressão, entre outras, que são as principais causas de incapacidade e mortalidade entre os idosos (SILVA et al., 2017).

Neste sentido, uma expressiva parte dos idosos torna-se mais sensível e, por necessitar de cuidados específicos, a família ou pessoas próximas, muitas vezes, recorrem às instituições de longa permanência para idosos (ILPI), locais destinados àqueles que não possuem cuidado estruturado dentro do âmbito familiar ou residem sozinhos (SICILIA et al., 2015).

Em consequência dos aspectos supracitados, o consumo de medicamentos aumenta na população idosa e isto pode desencadear sérios problemas à saúde destes indivíduos, como, por exemplo, a polifarmácia, que é o uso de cinco ou mais medicamentos diariamente (SI-
CILIA et al., 2015; BAZARGAN et al., 2017). Dentre os medicamentos administrado que podem estar incluídos no âmbito da polifarmácia, encontram-se os benzodiazepínicos, que são os psicotrópicos mais utilizados para funções ansiolítica, hipnótica, anticonvulsivante, relaxante muscular e atuar sobre os quadros de ansiedade, insônia e epilepsia. Entretanto, são drogas que alteram os aspectos cognitivos e psicomotores do organismo (SICILIA et al., 2015).

No Brasil, há uma escassez na literatura de estudos relacionados à farmacoterapia aplicada em idosos institucionalizados, sendo este tema amplamente abordado na comunidade e no âmbito hospitalar (SMANIOTO; HADDAD, 2013; ASSIS et al., 2016). Com base nisto, o objetivo deste estudo foi verificar a prevalência de polifarmácia, benzodiazepínicos e fatores associados em idosos institucionalizados.

\section{Materiais e métodos}

Estudo transversal, de base populacional, que faz parte do projeto intitulado "Padrões de envelhecimento e longevidade: aspectos biológicos, educacionais e psicossociais de idosos institucionalizados", desenvolvido pelo Programa de Pós-Graduação em Envelhecimento Humano da Universidade de Passo Fundo.

O estudo foi realizado em dez ILPI privadas e filantrópicas localizadas nos municípios de Passo Fundo e Bento Gonçalves, no interior do Estado do Rio Grande do Sul. Foram incluídos, os indivíduos com 60 anos ou mais, de 
ambos os sexos, independente do tempo de residência na instituição. Os critérios de exclusão estabelecidos foram para indivíduos que estivessem hospitalizados no dia do encontro com a equipe de pesquisa.

A coleta de dados iniciou logo após a assinatura do Termo de Consentimento Livre e Esclarecido pelos idosos ou responsáveis. Os entrevistadores foram recrutados nos cursos de graduação e pós-graduação da Universidade de Passo Fundo, os quais receberam treinamento prévio. Coletaram-se os dados por meio de um questionário estruturado composto por 23 blocos. No presente estudo, foram utilizadas as variáveis dos blocos A (dados de identificação), B (variáveis sociodemográficas), C (estado cognitivo) e G (condições de saúde).

As informações dos blocos A e B foram coletadas por meio de entrevista com os responsáveis. Para avaliar o estado cognitivo dos idosos (bloco $\mathrm{C}$ ), foi utilizado Mini Exame de Estado Mental, cujos escores adotados levaram em consideração o nível de escolaridade para analfabeto (13 pontos), indivíduos com 1 a 8 anos de estudo (18 pontos) e indivíduos com 9 anos ou mais de estudo (26 pontos) (BERTOLUCCI et al., 1994). As variáveis referentes às condições de saúde (bloco G) foram coletadas por meio de consulta ao prontuário dos residentes. Os medicamentos foram classificados por grupos farmacológicos de acordo com o Anatomical Therapeutic Chemical, desenvolvida e adotada pela World Health Organization Collaborating Centre for Drug Statistics Methodology (2017). Os dados foram codificados e armazenados em um banco de dados no software estatístico.

Para análise empregou-se estatística descritiva e inferencial. As variáveis qualitativas (categóricas, nominais e ordinais) foram apresentadas empregando-se distribuições de frequências univariadas (absolutas e relativas). Para avaliar a associação entre as variáveis categóricas, aplicaram-se os testes Qui-Quadrado. O nível de significância adotado foi de $5 \%$.

Este estudo foi aprovado pelo Comitê de Ética e Pesquisa em Seres Humano da Universidade de Passo Fundo sob parecer número 2.097.278, e está de acordo com as constatações éticas da Declaração de Helsinki e as normas constantes na Resolução 466/2012 do Conselho Nacional de Saúde.

\section{Resultados}

Ao todo, 219 idosos compuseram a amostra do presente estudo. A Tabela 1 apresenta a caracterização do perfil sociodemográfico dos idosos institucionalizados. 
Tabela 1. Caracterização do perfil sociodemográfico dos idosos institucionalizados. Passo Fundo e Bento Gonçalves, Rio Grande do Sul, Brasil, 2017.

\begin{tabular}{|c|c|c|}
\hline Variáveis/Categorias & $\mathbf{n}$ & $\%$ \\
\hline \multicolumn{3}{|l|}{ Sexo } \\
\hline Feminino & 143 & 65,3 \\
\hline Masculino & 76 & 34,7 \\
\hline \multicolumn{3}{|l|}{ Faixa etária } \\
\hline 60-69 anos & 28 & 12,8 \\
\hline 70-79 anos & 64 & 29,2 \\
\hline$\geq 80$ anos & 127 & 58,0 \\
\hline \multicolumn{3}{|l|}{ Escolaridade } \\
\hline Analfabeto & 42 & 19,2 \\
\hline 1 à 8 anos de estudo & 147 & 67,1 \\
\hline 9 anos de estudo ou mais & 26 & 11,9 \\
\hline Não respondeu & 4 & 1,8 \\
\hline \multicolumn{3}{|l|}{ Cor } \\
\hline Branca & 189 & 86,3 \\
\hline Preta & 9 & 4,1 \\
\hline Parda & 14 & 6,4 \\
\hline Amarela & 2 & 0,9 \\
\hline Não respondeu & 5 & 2,3 \\
\hline \multicolumn{3}{|l|}{ Estado civil } \\
\hline Solteiro & 59 & 26,9 \\
\hline $\begin{array}{l}\text { Casado (a) / Com companheiro } \\
\text { (a) }\end{array}$ & 13 & 5,9 \\
\hline Viúvo (a) & 113 & 51,6 \\
\hline Divorciado (a) / Separado (a) & 33 & 15,1 \\
\hline Não respondeu & 1 & 0,5 \\
\hline \multicolumn{3}{|l|}{ Condição cognitiva } \\
\hline Com declínio cognitivo & 158 & 72,1 \\
\hline Sem declínio cognitivo & 61 & 27,9 \\
\hline
\end{tabular}

Observou-se que a maioria da amostra era composta por mulheres, com idade igual ou superior aos 80 anos de idade, com escolaridade de 1 à 8 anos de estudo, brancas, viúvas e com declínio cognitivo. A Tabela 2 apresenta a ca- racterização das condições de saúde dos idosos estudados. 
Tabela 2. Condições de saúde dos idosos institucionalizados. Passo Fundo e Bento Gonçalves, Rio Grande do Sul, Brasil, 2017.

\begin{tabular}{|l|c|c|}
\hline \multicolumn{1}{|c|}{ Variáveis/Categorias } & $\mathbf{n}$ & $\%$ \\
\hline Hipertensão arterial sistêmica & 113 & 53,8 \\
\hline Demência & 100 & 47,6 \\
\hline Dor crônica & 70 & 34,8 \\
\hline Insônia & 69 & 33,0 \\
\hline Depressão & 68 & 32,7 \\
\hline Diabetes mellitus & 46 & 21,7 \\
\hline Acidente vascular encefálico & 43 & 20,3 \\
\hline Reumatismo & 40 & 18,9 \\
\hline Doença cardiovascular & 33 & 15,8 \\
\hline Osteoporose & 28 & 13,2 \\
\hline Parkinson & 19 & 9,0 \\
\hline Doença pulmonar & 12 & 5,7 \\
\hline Outras morbidades & 59 & 28,5 \\
\hline
\end{tabular}

Constatou-se que problemas de saú- taram um número expressivo de acode que apresentaram maior prevalência metimento, como a dor crônica, insônia foram a hipertensão arterial sistêmica e depressão, respectivamente. A Tabela e a demência, respectivamente. Outros 3 apresenta o perfil medicamentoso dos problemas de saúde também apresen- idosos institucionalizados.

Tabela 3. Perfil medicamentoso dos idosos institucionalizados. Passo Fundo e Bento Gonçalves, Rio Grande do Sul, Brasil, 2017.

\begin{tabular}{|l|c|c|}
\hline \multicolumn{1}{|c|}{ Variáveis/Categorias } & $\mathbf{n}$ & $\%$ \\
\hline Polifarmácia & 155 & 74,5 \\
\hline Cardiovasculares & 145 & 71,9 \\
\hline Gastrointestinais & 106 & 53,3 \\
\hline Suplementos vitamínicos & 103 & 51,8 \\
\hline Antipsicóticos & 96 & 48,2 \\
\hline Antidepressivos & 93 & 46,2 \\
\hline Analgésicos/AINES & 85 & 42,1 \\
\hline Diuréticos & 71 & 35,7 \\
\hline $\begin{array}{l}\text { Antiparkinsonianos/Doença de } \\
\text { Alzheimer }\end{array}$ & 58 & 29,1 \\
\hline Repositor hormonal & 44 & 22,1 \\
\hline Benzodiazepínicos & 42 & 21,1 \\
\hline
\end{tabular}




\begin{tabular}{|l|l|l|}
\hline Antidiabéticos & 40 & 20,1 \\
\hline Outros & 92 & 46,0 \\
\hline
\end{tabular}

Legenda: AINES: Anti-inflamatórios não esteroides.

Duzentos e onze idosos $(97,7 \%)$ faziam uso de medicamentos receitados pelo médico nos últimos três meses. A prevalência de polifarmácia foi de 74,5\%, sendo que a média de medicamentos que os idosos faziam uso no momento da entrevista foi de 7,0 $\pm 3,5$ (zero a 22 medicamentos utilizados). Ainda, $53,5 \%$ dos idosos faziam uso de sete ou mais medicamentos.
Dentre as classes mais recorrentes observou-se que os medicamentos cardiovasculares foram os mais utilizados, seguido dos gastrointestinais, suplementos vitamínicos, antipsicóticos e antidepressivos. O uso de benzodiazepínicos foi de $21,1 \%$ da amostra analisada. A Tabela 4 apresenta a relação entre ocorrência de polifarmácia e as condições de saúde dos idosos institucionalizados.

Tabela 4. Relação entre a polifarmácia e as condições de saúde dos idosos institucionalizados. Passo Fundo e Bento Gonçalves, Rio Grande do Sul, Brasil, 2017..

\begin{tabular}{|c|c|c|c|c|}
\hline \multirow[t]{2}{*}{ Variáveis } & \multirow[t]{2}{*}{ Categorias } & \multicolumn{2}{|c|}{ Polifarmácia } & \multirow[t]{2}{*}{$p$} \\
\hline & & Sim & Não & \\
\hline \multirow[t]{2}{*}{ Função cognitiva } & Com declínio & $78(78,0 \%)$ & $22(22,0 \%)$ & \multirow[t]{2}{*}{0,651} \\
\hline & Sem declínio & $47(81,0 \%)$ & $11(19,0 \%)$ & \\
\hline \multirow[t]{2}{*}{ Hipertensão arterial sistêmica } & Sim & $92(83,6 \%)$ & $18(16,6 \%)$ & \multirow[t]{2}{*}{$<0,001^{*}$} \\
\hline & Não & $54(60,7 \%)$ & $35(39,3 \%)$ & \\
\hline \multirow[t]{2}{*}{ Demência } & Sim & $64(66,7 \%)$ & $32(33,3 \%)$ & \multirow[t]{2}{*}{$0,028^{*}$} \\
\hline & Não & $82(80,4 \%)$ & $20(19,6 \%)$ & \\
\hline \multirow[t]{2}{*}{ Dor crônica } & Sim & $52(80,0 \%)$ & $13(20,0 \%)$ & \multirow[t]{2}{*}{0,114} \\
\hline & Não & $88(69,3 \%)$ & $39(30,7 \%)$ & \\
\hline \multirow[t]{2}{*}{ Insônia } & Sim & $47(73,4 \%)$ & $17(26,6 \%)$ & \multirow[t]{2}{*}{0,917} \\
\hline & Não & $98(73,7 \%)$ & $35(26,3 \%)$ & \\
\hline \multirow[t]{2}{*}{ Depressão } & Sim & $57(86,4 \%)$ & $9(13,6 \%)$ & \multirow[t]{2}{*}{$0,004^{*}$} \\
\hline & Não & $88(67,2 \%)$ & $43(32,8 \%)$ & \\
\hline \multirow[t]{2}{*}{ Diabetes mellitus } & Sim & $36(83,7 \%)$ & $7(16,3 \%)$ & \multirow[t]{2}{*}{0,090} \\
\hline & Não & $112(70,9 \%)$ & $6(29,1 \%)$ & \\
\hline \multirow[t]{2}{*}{ Acidente vascular encefálico } & Sim & $28(73,7 \%)$ & $10(26,3 \%)$ & \multirow[t]{2}{*}{0,977} \\
\hline & Não & $119(73,5 \%)$ & $43(26,5 \%)$ & \\
\hline \multirow[t]{2}{*}{ Neoplasia } & Sim & $6(60,0 \%)$ & $4(40,0 \%)$ & \multirow[t]{2}{*}{0,316} \\
\hline & Não & $142(74,3 \%)$ & $49(25,7 \%)$ & \\
\hline
\end{tabular}




\begin{tabular}{|l|l|l|l|c|}
\hline \multirow{2}{*}{ Reumatismo } & Sim & $30(76,9 \%)$ & $9(23,1 \%)$ & \multirow{2}{*}{0,603} \\
\cline { 2 - 4 } & Não & $118(72,8 \%)$ & $44(27,2 \%)$ & \\
\hline \multirow{2}{*}{ Doença cardiovascular } & Sim & $30(93,8 \%)$ & $2(6,3 \%)$ & \multirow{2}{*}{$0,005^{*}$} \\
\cline { 2 - 4 } & Não & $116(69,9 \%)$ & $50(30,1 \%)$ & \\
\hline \multirow{2}{*}{ Osteoporose } & Sim & $24(85,7 \%)$ & $4(14,3 \%)$ & \multirow{2}{*}{0,118} \\
\cline { 2 - 4 } & Não & $124(71,7 \%)$ & $49(28,3 \%)$ & \\
\hline Parkinson & Sim & $15(78,9 \%)$ & $4(21,1 \%)$ & \multirow{2}{*}{0,563} \\
\cline { 2 - 4 } & Não & $131(72,8 \%)$ & $49(27,2 \%)$ & \multirow{2}{*}{0,420} \\
\hline \multirow{2}{*}{ Doença pulmonar } & Sim & $10(83,3 \%)$ & $2(16,7 \%)$ & \\
\cline { 2 - 4 } & Não & $136(72,7 \%)$ & $51(27,3 \%)$ & \\
\hline
\end{tabular}

Legenda: *significância estatística para $\mathrm{p}<0,05$.

As análises evidenciaram que houve cardiovasculares $(\mathrm{p}=0,005)$. A Tabela 5 relação significativa entre a ocorrência apresenta a relação entre os benzodiade polifarmácia e a hipertensão arterial zepínicos e as condições de saúde dos sistêmica $(\mathrm{p}<0,001)$, demência $(\mathrm{p}=$ idosos institucionalizados. $0,028)$, depressão $(\mathrm{p}=0,004)$ e doenças

Tabela 5. Relação entre o consumo de benzodiazepínicos e as condições de saúde dos idosos institucionalizados. Passo Fundo e Bento Gonçalves, Rio Grande do Sul, Brasil, 2017.

\begin{tabular}{|c|c|c|c|c|}
\hline \multirow[t]{2}{*}{ Variáveis } & \multirow[t]{2}{*}{ Categorias } & \multicolumn{2}{|c|}{ Benzodiazepínicos } & \multirow[t]{2}{*}{$p$} \\
\hline & & Sim & Não & \\
\hline \multirow[t]{2}{*}{ Função cognitiva } & Com declínio & $22(22,4 \%)$ & $76(77,6 \%)$ & \multirow[t]{2}{*}{0,533} \\
\hline & Sem declínio & $10(18,2 \%)$ & $45(81,8 \%)$ & \\
\hline \multirow[t]{2}{*}{ Hipertensão arterial sistêmica } & Sim & $20(18,9 \%)$ & $86(81,1 \%)$ & \multirow[t]{2}{*}{0,661} \\
\hline & Não & $18(21,4 \%)$ & $66(78,6 \%)$ & \\
\hline \multirow[t]{2}{*}{ Demência } & Sim & $12(13,5 \%)$ & $77(86,5 \%)$ & \multirow[t]{2}{*}{$0,022^{*}$} \\
\hline & Não & $27(27,0 \%)$ & $73(73,0 \%)$ & \\
\hline \multirow[t]{2}{*}{ Dor crônica } & Sim & $14(22,2 \%)$ & $49(77,8 \%)$ & \multirow[t]{2}{*}{0,114} \\
\hline & Não & $23(19,2 \%)$ & $97(80,8 \%)$ & \\
\hline \multirow[t]{2}{*}{ Insônia } & Sim & $15(24,6 \%)$ & $46(75,4 \%)$ & \multirow[t]{2}{*}{0,367} \\
\hline & Não & $24(18,9 \%)$ & $103(81,1 \%)$ & \\
\hline \multirow[t]{2}{*}{ Depressão } & Sim & $16(25,0 \%)$ & $48(75,0 \%)$ & \multirow[t]{2}{*}{0,240} \\
\hline & Não & $22(17,7 \%)$ & $102(82,3 \%)$ & \\
\hline \multirow[t]{2}{*}{ Diabetes mellitus } & Sim & $5(12,5 \%)$ & $35(87,5 \%)$ & \multirow[t]{2}{*}{0,167} \\
\hline & Não & $34(22,4 \%)$ & $118(77,6 \%)$ & \\
\hline \multirow[t]{2}{*}{ Acidente vascular encefálico } & Sim & $3(7,9 \%)$ & $35(92,1 \%)$ & \multirow[t]{2}{*}{$0,032^{\star}$} \\
\hline & Não & $36(23,5 \%)$ & $117(76,5 \%)$ & \\
\hline
\end{tabular}




\begin{tabular}{|c|c|c|c|c|}
\hline \multirow[t]{2}{*}{ Neoplasia } & Sim & $2(20,0 \%)$ & $8(80,0 \%)$ & \multirow[t]{2}{*}{0,980} \\
\hline & Não & $37(20,3 \%)$ & $145(79,7 \%)$ & \\
\hline \multirow[t]{2}{*}{ Reumatismo } & Sim & $9(24,3 \%)$ & $28(75,7 \%)$ & \multirow[t]{2}{*}{0,500} \\
\hline & Não & $30(19,4 \%)$ & $125(80,6 \%)$ & \\
\hline \multirow[t]{2}{*}{ Doença cardiovascular } & Sim & $6(19,4 \%)$ & $25(80,6 \%)$ & \multirow[t]{2}{*}{0,909} \\
\hline & Não & $32(20,3 \%)$ & $126(79,7 \%)$ & \\
\hline \multirow[t]{2}{*}{ Osteoporose } & Sim & $9(32,1 \%)$ & $19(67,9 \%)$ & \multirow[t]{2}{*}{0,090} \\
\hline & Não & $30(18,3 \%)$ & $134(81,7 \%)$ & \\
\hline \multirow[t]{2}{*}{ Parkinson } & Sim & $4(22,2 \%)$ & $14(77,8 \%)$ & \multirow[t]{2}{*}{0,851} \\
\hline & Não & $35(20,3 \%)$ & $137(79,7 \%)$ & \\
\hline \multirow[t]{2}{*}{ Doença pulmonar } & Sim & $2(16,7 \%)$ & $10(83,3 \%)$ & \multirow[t]{2}{*}{0,800} \\
\hline & Não & $35(19,7 \%)$ & $143(80,3 \%)$ & \\
\hline
\end{tabular}

Constatou-se que houve uma associação entre o não uso de benzodiazepínicos e o acidente vascular encefálico ( $\mathrm{p}$ $=0,032)$ e a demência $(\mathrm{p}=0,022)$.

\section{Discussão}

O perfil da amostra era predominantemente feminino, longevo, branco, em condição de viuvez, com escolaridade básica e com declínio cognitivo, o que corrobora com um estudo que caracterizou o perfil de idosos institucionalizados em seis cidades gaúchas e evidenciou que a maioria dos residentes era composta por mulheres $(62,9 \%)$, com baixa ou nenhuma escolaridade e com 80 anos ou mais (48,3\%) (ROSA et al., 2011). Outro estudo apontou que o declínio cognitivo em idosos institucionalizados chegou a $81,2 \%$ (LENI; PORTELLA; DORING, 2016).

Segundo a Organização Pan-Americana da Saúde (2005), os principais problemas de saúde enfrentados pelos idosos são as doenças cardiovasculares, a hipertensão arterial sistêmica, o acidente vascular encefálico, o diabetes mellitus, as neoplasias, as doenças pulmonares, as doenças musculoesqueléticas, a demência, as doenças mentais e a depressão. Observou-se neste estudo a ocorrência de todas as doenças supracitadas, sendo que a hipertensão arterial sistêmica foi a mais prevalente, o que vai ao encontro do estudo de Dantas et al. (2013), que encontrou uma prevalência desta doença em 48,8\% em idosos institucionalizados.

Os idosos expostos ao risco de desenvolver doenças crônicas tornam-se grandes consumidores de medicamentos e, muitas vezes, de um número muito mais elevado que o necessário, possibilitando o aumento das chances de ocorrer reações adversas e interações medicamentosas. Os idosos residentes em ILPI estão mais propensos à esta problemática (SALES; SALES; CASOTTI, 2017).

A institucionalização pode condicionar os idosos à polifarmácia e seus eventos adversos, muitas vezes, em consequência de doenças limitantes, 
fragilidade e restrição da funcionalidade (LUCCHETTI et al., 2010). Neste estudo, 74,5\% dos idosos institucionalizados faziam uso de polifarmácia, o que corrobora com o estudo de Reis e Jesus (2017), onde a proporção de idosos com a mesma problemática foi de $69,7 \%$.

Uma série de eventos adversos pode ser desencadeada por conta da polifarmácia, como reações adversas às interações medicamentosas, menor adesão à terapia, diminuição da capacidade funcional e do declínio cognitivo (ALMEIDA et al., 2017). No presente estudo observou-se associação entre a polifarmácia e as doenças cardiovasculares, a hipertensão arterial sistêmica, a depressão e a demência. Informações semelhantes foram encontradas no estudo de Jiménez et al. (2017).

Outros estudos apontam para a associação entre a polifarmácia e doenças crônicas nos idosos, como a demência (LUCCHETTI et al., 2010), a depressão (BARBOSA, 2015) e doenças do aparelho circulatório, como é o caso da hipertensão arterial sistêmica (ALMEIDA et al., 2017). Além disso, a associação entre a polifarmácia e as doenças cardiovasculares nos idosos do presente estudo, pode-se ser justificada pela necessidade de associações medicamentosas para o tratamento de múltiplas doenças do sistema cardiovascular como arritmias, dislipidemia, síndrome metabólica e a própria hipertensão arterial sistêmica (LUCCHETTI et al., 2010).

Em geral, os idosos que fazem uso de benzodiazepínicos justificam isso como sendo de uso paliativo para lidar com suas dificuldades existenciais, culturais, sociais e familiares (ALVARENGA et al., 2015). No Brasil, o consumo de benzodiazepínicos varia entre 22 e $30 \%$ de acordo com a literatura ou o contexto ao qual o idoso está inserido (ALVARENGA et al., 2007; NORDON et al., 2009), podendo este número chegar à $61,4 \%$ nas ILPI (GÓMEZ et al., 2017). Embora a prevalência de benzodiazepínicos encontrada neste estudo seja semelhante à média nacional, este número é menor ao encontrado em idosos institucionalizados $(21,1 \%)$. A literatura aponta que este é um valor alto, visto os efeitos adversos que os benzodiazepínicos podem causar a saúde dos seus usuários (ALVIM et al., 2017).

A prevalência de benzodiazepínicos encontrados nos idosos institucionalizados assemelham-se a prevalência de benzodiazepínicos encontrados em idosos da comunidade (ALVIM et al., 2017), porém não encontramos associações entre o seu uso e as variáveis independentes, corroborando com o estudo de Wang et al (2017), onde não foram constatadas relação entre o uso de benzodiazepínicos e as condições de saúde.

Mediante as análises observadas, o presente estudo não está livre de apresentar limitações que pudessem influenciar os resultados obtidos. Acredita-se que o monitoramento do uso de benzodiazepínicos em idosos institucionalizados seja controlado e adequado para esta população (BICCA; ARGIMON, 2008), o que explicaria a prevalência de apenas $21,1 \%$ em nosso estudo. Além disso, esta explicação também pode justificar não 
terem sido encontradas associações entre o uso de benzodiazepínicos e as variáveis secundarias, mas sim a associação entre o não uso de benzodiazepínicos e a demência e o acidente vascular encefálico. Embora existam limitações, isso não inviabiliza a geração dos dados apresentados neste estudo.

Com o aumento do envelhecimento populacional brasileiro, do índice de doenças crônicas e do uso de medicamentos por estes indivíduos (ALMEIDA et al., 2017), os estudos de perfis têm prestado importantes informações a respeito do uso de medicamentos por idosos institucionalizados (OLIVEIRA; NOVAES, 2013; FOCHAT et al., 2012). Desta forma, o desempenho dos profissionais da saúde deve estar ligado diretamente ao conhecimento da polifarmácia e benzodiazepínicos entre os idosos, promovendo formas de gerenciar o plano terapêutico dos fármacos de uso crônico na pretensão de minimizar os efeitos adversos e evitar internações desnecessárias, incapacitação progressiva e perda da autonomia. Neste sentido, os achados deste estudo oferecem subsídios para a construção de políticas públicas que visem atenção à pessoa idosa institucionalizada.

\section{Conclusão}

A polifarmácia é altamente prevalente em idosos institucionalizados e relaciona-se a presença de hipertensão arterial sistêmica, demência, depressão e doenças cardiovasculares. $\mathrm{O}$ uso de benzodiazepínicos é expressivo nesta população, contudo seu uso não relaciona-se a desfechos secundários.

Prevalence of polypharmacy, benzodiazepines and associated factors in institutionalized elderly

\section{Abstract}

With human aging there is an increase in the health problems presented by the elderly, and often the family members, or the individuals themselves, opt for specialized geriatric care, such as long-term care for the elderly. Concomitant to this, there is an increase in the consumption of drugs by this population, especially benzodiazepines, thus characterizing polypharmacy. Based on this, the objective of this study was to verify the prevalence of polypharmacy, benzodiazepines and associated factors in institutionalized elderly. Cross-sectional, population-based study with 219 elderly residents in 10 long-term care for the elderly distributed in Passo Fundo/RS and Bento Gonçalves/RS. The data were collected through a structured questionnaire, where the identification data, sociodemographic variables, cognitive status and health conditions were recorded. For the analysis, descriptive and inferential statistics were used. It was observed that the majority of the sample consisted of women, white, 80 years old or older, low schooling, widows, with cognitive decline and with chronic diseases. The prevalence of polypharmacy reached $74.5 \%$ and of benzodiazepines $21.1 \%$. Polypharmacy was associated with systemic arterial hypertension, dementia, depression and cardiovascular diseases $(p<0.05)$, while benzodiazepines were inversely associated with cerebrovascular accident and dementia $(p<0.05)$. In short, the evidence presented can instigate the construction of public policies, seen in better health conditions and attention to 
polypharmacy in this population.

Keywords: Aging. Polypharmacy. Health status. Homes for the aged.

\section{Referências}

ALMEIDA, N. A. et al. Prevalence of and factors associated with polypharmacy among elderly persons resident in the community. Revista Brasileira de Geriatria e Gerontologia, Rio de Janeiro, v. 20, n. 1, p. 138-148, 2017.

ALVARENGA, J. M. et al. Prevalence and sociodemographic characteristics associated with benzodiazepines use among community dwelling older adults: The Bambuí Health and Aging Study (BHAS). Revista Brasileira de Psiquiatria, São Paulo, v. 1, n. 1, p. 7-11, 2007.

ALVARENGA, J. M. et al. Uso de benzodiazepínicos entre idosos: o alívio de "jogar água no fogo", não pensar e dormir. Revista Brasileira de Geriatria e Gerontologia, Rio de Janeiro, v. 18, n. 2, p. 249-258, 2015.

ALVIM, Mariana Macedo et al. Prevalência e fatores associados ao uso de benzodiazepínicos em idosos da comunidade. Revista Brasileira de Geriatria e Gerontologia, Rio de Janeiro, v. 20, n. 4, p. 463-473, 2017.

ASSIS, D. L. et al. Polifarmácia e uso de medicamentos inapropriados em idosos institucionalizados: lições ainda não aprendidas. Geriatrics, Gerontology and Aging, xx, v. 10, n. 3, p. 126-131, 2016.

BARBOSA, R. A. S. R. Qualidade de vida, suporte familiar, comorbidades e polifarmácia em idosos institucionalizados com e sem sintomas de depressão. Dissertação (Mestrado Acadêmico em Saúde e Sociedade) Faculdade de Enfermagem, Universidade do Estado do Rio Grande do Norte. Mossoró, 2015.

BAZARGAN, M. et al. Non-adherence to medication regimens among older African-
-American adults. BMC Geriatrics, Londres, v. 17, n. 1, p. 163, 2017.

BERTOLUCCI, P. H. F. et al. O Mini-Exame do Estado Mental em uma população geral: impacto da escolaridade. Arquivos de Neuro-Psiquiatria, São Paulo, v.m52, n. 1, p. 01-07, 1994.

BICCA, M. G.; ARGIMON, I. I. L. Cognitive skills and the use of benzodiazepinic drugs in institutionalized elderly women. Jornal Brasileiro de Psiquiatria, Rio de Janeiro, v. 57, n. 2, p. 133-138, 2008.

CAMARANO, A. A.; KANSO, S. As instituições de longa permanência para idosos no Brasil. Revista Brasileira de Estudos de População, São Paulo v. 27, n. 1, p. 232-235, 2010.

CRUZ, D. T. et al. Associação entre capacidade cognitiva e ocorrência de quedas em idosos. Cadernos de Saúde Coletiva, Rio de Janeiro, v. 23, n. 4, p. 386-393, 2015.

DANTAS, C. M. H. L. et al. Capacidade funcional de idosos com doenças crônicas residentes em Instituições de Longa Permanência. Revista Brasileira de Enfermagem, Brasília, v. 66, n. 6, p. 914-920, 2013.

GLENZ, F. et al. The elderly patient: no reason to worry!? Swiss Dental Journal, xx, v. 125 , n. 4 , p. 427- 431, 2015.

FOCHAT, R. C. et al. Perfil de utilização de medicamentos por idosos frágeis institucionalizados na Zona da Mata Mineira, Brasil. Revista de Ciências Farmacêuticas Básica e Aplicada, Araraquara, v. 33, n. 3, p. 447454, 2012.

GÓMEZ, S. et al. Uso de benzodiazepinas en adultos mayores en América Latina. Revista Médica de Chile, Santiago, v. 145, n. 3, p. 351-359, 2017.

JIMÉNEZ, R. M. R.; NAVARRO, C. O.; COMPÉS, C. C. La polifarmacia del paciente crónico complejo y la nutrición enteral. $\mathrm{Nu}$ trición Hospitalaria, Madrid, v. 34, supl.1, p. 57-76, 2017. 
LINI, E. V.; PORTELLA, M. R.; DORING, M. Fatores associados à institucionalização de idosos: estudo caso-controle. Revista Brasileira de Geriatria e Gerontologia, Rio de Janeiro, v. 19, n. 6, p. 1004-1014, 2016.

LUCCHETTI, G. et al. Fatores associados à polifarmácia em idosos institucionalizados. Revista Brasileira de Geriatria e Gerontologia, Rio de Janeiro, v. 13, n. 1, p. 51-58, 2010.

NORDON, D. G. et al. Características do uso de benzodiazepínicos por mulheres que buscavam tratamento na atenção primária. Revista de Psiquiatria do Rio Grande do Sul, Porto Alegre, v. 31, n. 3, p. 152-158, 2009.

OliveirA, M. P. F.; NOVAES, M. R. C. G. Perfil socioeconômico, epidemiológico e farmacoterapêutico de idosos institucionalizados de Brasília, Brasil. Ciência e Saúde Coletiva, Rio de Janeiro, v. 18, n. 4, p. 10691078, 2013.

ORGANIZAÇÃO PAN-AMERICANA DA SAÚDE. Envelhecimento ativo: uma política de saúde. Brasília, p. 62, 2005. Disponível em: <http://bvsms.saude.gov.br/bvs/publicacoes/envelhecimento_ativo.pdf $>$. Acesso em: 19 de outubro de 2017.

PRESTA, S. A. et al. Caracterização e condições de saúde dos idosos do município de Passo Fundo, no Rio Grande do Sul. Revista Brasileira de Ciências da Saúde, São Caetano do Sul, v. 9, n. 29, p. 31-38, 2011.

REIS, K. M. C.; JESUS, C. A. C. Relationship of polypharmacy and polypathology with falls among institutionalized elderly. Texto \& Contexto - Enfermagem, Florianópolis, v. 26, n. 2, p. e03040015, 2017.

ROSA, P. V. et al. Perfil dos idosos residentes em instituições de longa permanência da região sul do país. Revista Brasileira de Ciências do Envelhecimento Humano, Passo Fundo, v. 8, n. 1, p. 38-47, 2012.

SALES, A. S.; SALES, M. G. S.; CASOTTI, C. A. Perfil farmacoterapêutico e fatores associados à polifarmácia entre idosos de Aiquara, Bahia, em 2014*. Epidemiologia e
Serviços Saúde, Brasília, v. 26, n. 1, p. 121132, 2017.

SICILIA, A. B. G. et al. Analisis de la prescripción farmacológica en pacientes institucionalizados. Semergen - Medicina de Familia, Barcelona, v. 41, n. 8, p. 413-420, 2015.

SILVA, J. et al. Impact of insomnia on self-perceived health in the elderly. Arquivos de Neuro-psiquiatria, São Paulo, v. 75, n. 5, p. 277-281, 2017.

SMANIOTO, F. N.; HADDAD, M. C. L. Avaliação da farmacoterapia prescrita a idosos institucionalizados. Revista Brasileira de Enfermagem, Brasília, v. 66, n. 4, p. 523527, 2013.

WANG, M. T. et al. Benzodiazepine and Z-drug use and risk of pneumonia in patients with chronic kidney disease: A population-based nested case-control study. PLoS One, São Francisco, v. 12, n. 7, p. e0179472, 2017.

WORLD HEALTH ORGANIZATION COLLABORATING CENTRE FOR DRUG STATISTICS METHODOLOGY. ATC / DDD Index 2018. Oslo, Noruega. 2017. Disponível em: < https://www.whocc.no/atc_ddd_index/>. Acesso em: 29 de dezembro de 2017. 